BULL. AUSTRAL. MATH. SOC.

VOL. 33 (1986), 449-455

\title{
ORTHOGONALITY IN NORMED SPACES
}

\author{
J.R. PARTINGTON
}

\begin{abstract}
Some properties which different definitions or orthogonality in a normed space can possess are considered. It is shown that orthogonality can be defined on any separable space with many of the properties possessed by the usual orthogonality in an inner-product space, but that the possession of a further property forces the space to be isomorphic to a Euclidean space.
\end{abstract}

The orthogonality of two vectors in a Euclidean normed space can be characterized in numerous different ways. Several of these definitions of orthogonality have been applied in general normed spaces, giving, in general, distinct notions of orthogonality each with various convenient properties. However, no obviously "best possible" notion of orthogonality has been produced so far: it is our intention here to investigate what properties orthogonality relations may have in a general normed space which is not necessarily Euclidean. All our normed spaces will be over the reals.

The most commonly used definition of orthogonality is Birkhoff-Jomes orthogonality: if $x$ and $y$ are elements of a nomed space $X$, we say that $x+y$ (BJ) if and only if $\|x+\lambda y\| \geq\|x\|$ for all scalars $\lambda$ (see Diestel [2].)

A more recent definition is due to Diminnie [3]: $x \perp y$ (D) if and only if

$$
\sup \left\{\left|\begin{array}{ll}
f(x) & f(y) \\
g(x) & g(y)
\end{array}\right|: f, g, \in X^{*},\left\|f^{\|},\right\| g^{\sharp} \leq 1\right\}=\|x\| \Downarrow y \| .
$$

Received 27 August 1985. I am grateful to Professor Diminnie for his helpful comments.

Copyright Clearance Centre, Inc. Serial-fee code: 0004-9727/86 $\$ A 2.00+0.00$. 
Two older notions given by James [5] are Isosceles Orthogonality: $x \perp y$ (I) if and only if $\|x+y\|=\|x-y\|$, and Pythagorean Orthogonality: $x+y(\mathrm{P})$ if and only if $\left\|x^{2}\right\|+\|y\|^{2}=\|x-y\|^{2}$.

We shall be interested in the following properties that a definition of orthogonality may possess in a normed linear space, properties that are always possessed by the usual notion of orthogonality in a Euclidean space.

(1) (Nondegeneracy):

(2) (Symmetry):

(3) (Homogeneity):

(4) ((Right-)Additivity):

(5) (Resolvability):

that $x \perp(a x+y)$;

(6) (Continuity): then $x \perp y$;

It has been observed [3] that if an orthogonality relation $\perp$ is nondegenerate, homogeneous and additive, then if $x \neq 0$ the number $a$ in (5) is unique when it exists. If an orthogonality is homogeneous and additive, then the set of vectors $y$ such that $x \perp y$ forms a linear subspace. Moreover, for an orthogonality satisfying (1), (3), (4) and (5), given $x \in X$ of norm $I$ we may define linear functional $f_{x} \in X^{\star}$, by

$$
f_{x}(y)=a \text {, where } y=a x+z \text { and } x \perp z \text {. }
$$

Thus $\operatorname{Ker}\left(f_{x}\right)=\{z: x \perp z\}$ and $f_{x}(x)=1$. One may naturally extend the definition to define $f_{\lambda x}=\lambda f_{x}$ for general scalars $\lambda$, when $\|x\|=1$, since $f_{-x}=-f_{x}$ already.

LEMMA 1. If $\perp$ is an orthogonality relation satisfying (1), (3), (4), (5) and (6), and $\|x\|=1$, then $f_{x}$ is continuous and the map taking $x$ to $f_{x}$ is noxm-weak* continuous on the conit sphere of $X$.

Proof. If $f_{x}$ is discontinuous, then there exist vectors $\left(y_{n}\right)$ of norm 1 with $y_{n}=\lambda_{n} x+x_{n}$, where the $\lambda_{n}$ are scalars tending to infinity in modulus and $x \perp x_{n}$ for each $n$. But that implies that $x \perp x_{n} / \lambda_{n}=y_{n} / \lambda_{n}-x \rightarrow-x$. Hence $x \perp-x$, a contradiction since $x \neq 0$. 
Suppose now that $x_{n} \rightarrow x$ and $f_{x_{n}}(y)=a_{n}$, where $x$ and each $x_{n}$ have norm 1. Then $x_{n} \perp\left(y-a_{n} x_{n}\right)$ for each $n$. If $\left(a_{n}\right)$ (or a subsequence) tend to infinity in modulus, then we have $x_{n}+y / a_{n}-x_{n}$, and hence, letting $n \rightarrow \infty$ and using (6), that $x \perp-x$, a contradiction. If $a_{n}$ accumulates at $a$, we have $x_{n}+y-a_{n} x_{n}$, so that $x \perp y-a x$ and $f_{x}(y)=a$; thus, since $\left(a_{n}\right)$ can only accumulate at one point the result follows and $a_{n} \rightarrow a=f_{x}(y)$.

Although BJ-orthogonality and D-orthogonality satisfy several of the conditions above, one cannot expect all to be satisfied in general, as the following result (given by Diminnie [3]) shows.

PROPOSITION 2. Let $X$ be a space of dimension at least 3. Then the following conditions are equivalent.

(i) $X$ is an inner-product space;

(ii) BJ-orthogonality is symmetric;

(iii) BJ-orthogonality is equivalent to D-orthogonality;

(iv) D-orthogonality is additive.

Despite the above result, it is often still possible to give a definition of orthogonality satisfying all the above conditions.

THEOREM 3. In any Banach space $X$ with a countable total sequence $\left(f_{n}\right) \in X^{*}$ it is possible to define an orthogonality relation satisfying (1), ..., (6) above.

Proof. We may assume, without loss of generality, that each $f_{n}$ has norm 1. The orthogonality is defined by saying that $x \perp y$ if and only if $\Sigma f_{n}(x) f_{n}(y) / n^{2}=0$.

Conditions (1) to (6) are easily verified for this relation.

If $X$ is separable, a result of ovsepian and Pelczynski [9] (see also Lindenstrauss and Tzafrixi [8]) states that $X$ has a bounded fundamental and total biorthogonal sequence $\left(x_{n}, f_{n}\right)$. In this case the orthogonality given by the above formula has the additional property that the $\left(x_{n}\right)$ are mutually orthogonal. It is not clear whether one can define an orthogonality relation with the above properties in a general Banach space. However, as 
Diminnie has observed, the existence of a continuous Euclidean norm $\|\cdot\| \|_{e}$ on $X$ is another sufficient condition.

Given that each $f_{x}$ is bounded and the map taking $x$ to $f_{x}$ is normweak* continuous when an orthogonality relation satisfies (1), .., (6), one might hope that there would be a uniform bound on the norm of $f_{x}$ as $x$ ranges over the unit sphere. Thus $\perp$ might satisfy

(7) (Boundectness): There is a constant $C>0$ such that if $x \perp z$ then $\|a x+z\| \geq\|x\|$ whenever $|a| \geq C$.

If $\perp$ satisfies (1), .., (6) then boundedness is equivalent to the following condition.

$\left(7^{\prime}\right)$ : There is a constant $C>0$ such that $\left\|f_{x}\right\| \leq C$ whenever $\|x\|=1$.

Both (BJ) -orthogonality and D-orthogonality satisfy (7), although neither satisfies all of the earlier conditions, in general. Unfortunately, the addition of (7) to conditions (1) to (6) restricts the underlying normed space dramatically.

THEOREM 4. If $X$ is a Bonach space and 1 an orthogonality relation satisfying (1), ..., (7), then $X$ is isomorphic to an inner-product space.

Proof. We shall adapt the proof of Theorem 2 of James [6], which states that if $\mathrm{BJ}$-orthogonality is left additive and $\operatorname{dim} X \geq 3$, then $X$ is an inner-product space.

Let $Y$ be a finite-dimensional subspace of $X$ with a normalized basis $x_{1}, \ldots, x_{n}$ and let $M=\operatorname{Ker} f_{x_{1}} \cap \ldots n \operatorname{Ker} f_{x_{n}}$. Any element of $y$ is orthogonal to the whole of $M$ and given $x \in X$ we may write $x=P(x)+z$ with $P(x) \in Y$ and $z \in M$. Condition (7) implies that $\|C(P(x)+x z)\| \geq\|P(x)\|$ and hence $\|P(x)\| \leq C$ l $x \|$. Therefore every finitedimensional subspace of $X$ is uniformly complemented in $X$. It now follows from results of Lindenstrauss and Tzafriri [7] that $X$ is isomorphic to an inner-product space.

Another natural condition to impose turns out to be even stronger and implies that $X$ is automatically an inner-product space.

(8) (Strong symmetry): If $\|x\|=\|y\|=1$ and $y-\mathrm{ax} \perp x$, for a scalar $a_{4}$ then $x-\alpha y \perp y$. 
Conditions (1) to (5) together with (8) impl $\ddot{y}$ that $f_{x}(y)=f_{y}(x)$ for all $x$ and $y$.

THEOREM 5. For con orthogonality relation 1 satisfying (1) to (5) the following conditions are equivalent.

(i) $\perp$ is strongly symmetric;

(ii) the map taking $x$ to $f_{x}$ is Zinear;

(iii) $X$ is isometrically an inner-product space.

Proof. (i) $\Rightarrow(i i)$ : since (i) implies that $f_{x}(y)=f_{y}(x)$ and the map taking $x$ to $f_{y}(x)$ is linear, the result follows.

(ii) $\Rightarrow$ (iii): Let $x$ and $y$ be any two nonzero vectors. Then $f_{r x+s y}(r x)=r^{2}\|x\|^{2}+r s f_{y}(x)$

and

$$
f_{r x+\varepsilon y}(s y)=r s f_{x}(y)+s^{2}\|y\|^{2} \text {. }
$$

Therefore $\|x x+s y\|^{2}=r^{2}\|x\|^{2}+s^{2}\|y\|^{2}+r s\left(f_{x}(y)+f_{y}(x)\right)$. Hence the intersection of the unit sphere of $x$ with any two-dimensional subspace is an ellipse, which implies that $X$ is isometrically an inner-product space (see, for example, Day [1]).

$$
\text { (iii) } \Rightarrow(i) \text { : immediate. }
$$

The equivalence of conditions (i) and (iii) is also contained in Lemma 1.4 of Freese, Diminnie and Andalafte [4], phrased there in terms of a function $v(x, y)$, which equals $-f_{x}(y)$ when $\|x\|=\|y\|=1$.

To conclude, we shall characterize all possible orthogonality relations on $\mathbb{R}^{2}$ satisfying (1) to (6) (and hence automatically (7)).

THEOREM 6. Let 1 be an orthogonality relation on $\mathbb{R}^{2}$ satisfying conditions (1) to (7). Then there exists a number $t, 0<t<\pi$, and $a$ monotonic fionction $f:[0, t] \rightarrow[t, \pi]$ such that two nonzero vectors $x=r(\cos \theta, \sin \theta)$ and $y=s(\cos \phi, \sin \phi)(r, s \in \mathbb{R}, 0 \leq \theta, \phi<\pi)$ are orthogonal if and only if either

(i) $0 \leq \theta \leq t$ and $f(\theta)=\phi$, or

(ii) $0 \leq \phi \leq t$ and $f(\phi)=\theta$.

Moreover every such function gives a well-defined orthogonality. 
Proof. Let us write $e_{\alpha}=(\cos \alpha, \sin \alpha)$. Clearly the orthogonality relation is determined by which $e_{\alpha}$ are orthogonal. For each $0 \leq x \leq \pi$ there is an $s \in[0, \pi)$ such that $e_{x} \perp e_{s}$. Let $t \in[0, \pi)$ be such that $e_{0} \perp e_{t}$. Then the function $s(x)(\bmod \pi)$ is continuous on $[0, \pi)$ and $s(x) \neq x$ for all $x$. Hence, since $s(x) \rightarrow 0$ or $\pi$ as $x \rightarrow t$ and $s(x)$ is also one-one on $[0, \pi)$, we have that $s(x)$ is monotonically increasing on $[0, t)$, mapping it to $[t, \pi)$, and then maps $[t, \pi]$ monotonically onto $[0, t]$, since $s(s(x))=x$. The definition of $f$ is now apparent, and the result follows. Conversely, given $f$, it is clear how to define $\perp$ satisfying (1), ..., (7).

\section{References}

[1] M.M. Day, Normed Zinear spaces (Springer-Verlag, Berlin-HeidelbergNew York, 3rd ed., 1973).

[2] J. Diestel, Geometry of Bcnach spaces (Springer-Verlag, BerlinHeidelberg-New York, 1975).

[3] C.R. Diminnie, "A new orthogonality relation for normed linear spaces", Math. Nachr. 114 (1983), 197-203.

[4] R.W. Freese, C.R. Diminnie and E.Z. Andalafte, "A study of generalized orthogonality relations in normed linear spaces", Math. Nachr. (to appear).

[5] R.C. James, "Orthogonality in normed linear spaces", Duke Math. J. 12 (1945), 291-302.

[6] R.C. James, "Inner products in normed spaces", Buzz. Amer. Math. Soc. 53 (1947), 559-566.

[7] J. Lindenstrauss and L. Tzafriri, "On the complemented subspaces problem", Israel J. Math. 9 (1971), 263-269.

[8] J. Lindenstrauss and L. Tzafriri, Classical Banach spaces I, (Springer-Verlag, Berlin-Heidelberg-New York, 1977). 
[9] R.I. Ovsepian and A. Pelczynski, "The existence in every separable Banach space of a fundamental total and bounded biorthogonal sequence and related constructions of uniformly bounded orthonormal systems in $L^{2}$ ", Studia Math. 54 (1975), 149-159.

Fitzwilliam College,

Cambridge University,

England, 